\title{
Swampland conjectures for type IIB orientifolds with closed-string $\mathrm{U}(1) \mathrm{s}$
}

\section{Martín Enríquez Rojo and Erik Plauschinn}

Arnold Sommerfeld Center for Theoretical Physics, Ludwig-Maximilians-Universität, Theresienstraße 37, 80333 München, Germany

E-mail: Martin.Enriquez@physik.uni-muenchen.de, e.plauschinn@uu.nl

ABSTRACT: We study the weak gravity conjecture, the swampland distance conjecture and the emergence proposal for $\mathcal{N}=1$ orientifold compactifications of type IIB string theory with O3-/O7-planes. We allow for orientifold projections with $h_{+}^{2,1} \neq 0$ which gives rise to closed-string $\mathrm{U}(1)$ gauge fields, and our findings show that certain structures present for $\mathcal{N}=2$ compactifications are not present for $\mathcal{N}=1$. In particular, assumptions about stability have to be relaxed and we encounter an ambiguity for the emergence of gauge symmetries associated with the $h_{+}^{2,1}$ sector.

KEYWORDS: D-branes, Supergravity Models

ArXiv EPrint: 2002.04050 


\section{Contents}

1 Introduction 1

2 Type IIB orientifolds 3

2.1 Calabi-Yau orientifolds 3

2.2 Effective action 5

3 D3-branes wrapping three-cycles $\quad 7$

3.1 D-branes 7

3.2 Stability 9

4 Swampland conjectures $\quad 11$

$\begin{array}{lll}4.1 & \text { Weak gravity conjecture } & 11\end{array}$

$\begin{array}{lll}4.2 & \text { Swampland distance conjecture } & 13\end{array}$

$\begin{array}{lll}4.3 & \text { Emergence proposal } & 16\end{array}$

$\begin{array}{llr}5 & \text { Summary and conclusions } & 19\end{array}$

\section{Introduction}

String theory is argued to be a consistent theory of quantum gravity including gauge interactions. As such, it provides an ideal testing ground for expectations a general quantumgravity theory should satisfy. However, conversely one can try to identify quantum-gravity features in string theory and conjecture them to hold in general - this is the approach known as the swampland program.

The swampland is defined as the set of consistent effective quantum field theories which cannot be completed into consistent theories of quantum gravity [1]. During the last years there has been an immense effort in pursuing the swampland program and in proposing and testing swampland conjectures. It is not possible to give an overview of the corresponding literature here, and we therefore want to refer to the review article [2]. There are however three conjectures which play a role for this paper: these are the weak gravity conjecture in its original form [3] and the weak gravity conjecture with scalar fields [4]; the swampland distance conjecture [5] in its refined version [6]; and the emergence proposal [7] which has been further developed in [2]. These conjectures will be stated in section 4 .

The swampland program typically does not rely on supersymmetry, but many computations in string theory are simplified considerably when some amount of supersymmetry is preserved. A well-established setting is that of type IIB string theory compactified on Calabi-Yau three-folds $\mathcal{X}$, for which the resulting theory in four dimensions is $\mathcal{N}=2$ supersymmetric. The massless spectrum is determined by the topology of the compact 
space, in particular, the four-dimensional theory contains one gravity multiplet, $h^{2,1}$ vector multiplets and $h^{1,1}+1$ hypermultiplets where $h^{2,1}$ and $h^{1,1}$ denote the non-trivial Hodge numbers of $\mathcal{X}$. In such compactifications one obtains a variety of BPS objects which have special properties and features, given for instance by D-branes wrapping cycles of the compact space. The authors of [7] consider such type IIB compactifications and focus on the vector-multiplet sector, which contains closed-string $\mathrm{U}(1)$ gauge fields and the complexstructure moduli of $\mathcal{X}$. The relevant BPS objects are D3-branes wrapping three-cycles of the Calabi-Yau manifold, and they are charged under the U(1)s and their mass depends on the complex-structure moduli. For this setting many explicit checks of various swampland conjectures have been performed and the inter-dependence of some of these conjectures has been illustrated.

The main question we want to ask in this paper is What happens to the $\mathcal{N}=2$ analysis when we perform an orientifold projection? It is well-known that under an orientifold projection (giving rise to O3- and O7-planes) the $h^{2,1}$ vector multiplets of $\mathcal{N}=2$ are projected to $h_{-}^{2,1}$ chiral multiplets and $h_{+}^{2,1}$ vector multiplets of four-dimensional $\mathcal{N}=1$ supergravity [8]. In particular, we have

\begin{tabular}{|c|c|c|}
\hline $\mathcal{N}=2$ & & $\mathcal{N}=1$ \\
\hline \multirow{2}{*}{$h^{2,1}$ vector multiplets } & orientifold & $h_{-}^{2,1}$ chiral multiplets \\
\hline & & $h_{+}^{2,1}$ vector multiplets \\
\hline
\end{tabular}

where $h^{2,1}=h_{-}^{2,1}+h_{+}^{2,1}$. Note that the $\mathcal{N}=1$ chiral multiplets contain the complexstructure moduli and the $\mathcal{N}=1$ vector multiplets contain the $\mathrm{U}(1)$ gauge fields, and that both multiplets are independent of each other. As we will argue, this implies that D3-branes wrapping three-cycles are no longer BPS but split into massive-uncharged and massless-charged states in the four-dimensional theory. Hence, a priori the analysis of [7] no longer applies.

We now want to briefly mention some works in the literature which are related to our discussion:

- As discussed above, the work of [7] studies swampland conjectures for $\mathcal{N}=2$ compactifications of type IIB string theory. Here we perform an analysis for the theory obtained after an orientifold projection to $\mathcal{N}=1$.

- In [9] the authors investigate (among others) domain walls in $\mathcal{N}=1$ orientifold compactifications of type II string theory. For the O3-/O7-situation, these domain walls are given by D5-branes wrapping three-cycles of the Calabi-Yau manifold. The mass of these four-dimensional states is determined by the complex-structure moduli and they are charged under closed-string three-form gauge fields, but not under closed-string one-form gauge fields.

This paper is organized as follows: in section 2 we review type IIB orientifold compactifications with orientifold three- and seven-planes, and in section 3 we analyze D3-branes 
wrapping three-cycles in the compact space. In section 4 we discuss the weak gravity conjecture, the swampland distance conjecture and the emergence proposal for this setting, and section 5 contains our summary and conclusions. We furthermore mention that this paper is based on the master thesis [10] of one of the authors, where further details and discussions can be found.

\section{Type IIB orientifolds}

In this section we briefly review Calabi-Yau orientifold compactifications of type IIB string theory with O3- and O7-planes. We consider the general situation with $h_{+}^{2,1} \neq 0$, leading to closed-string $\mathrm{U}(1)$ gauge fields in the four-dimensional effective theory [8].

\subsection{Calabi-Yau orientifolds}

We start by introducing our notation for the topology of Calabi-Yau orientifolds, and we provide some details on the special geometry of the complex-structure moduli space.

Topology. We consider a Calabi-Yau three-fold $\mathcal{X}$, which comes with a holomorphic three-form $\Omega \in H^{3,0}(\mathcal{X})$ and a real Kähler form $J \in H^{1,1}(\mathcal{X})$. To perform an orientifold projection we impose a holomorphic involution $\sigma$ on $\mathcal{X}$, chosen to act on $\Omega$ and $J$ as

$$
\sigma^{*} J=+J, \quad \sigma^{*} \Omega=-\Omega .
$$

Since $\sigma$ is an involution, the cohomology groups of the Calabi-Yau three-fold $\mathcal{X}$ split into even and odd eigenspaces as $H^{p, q}(\mathcal{X})=H_{+}^{p, q}(\mathcal{X}) \oplus H_{-}^{p, q}(\mathcal{X})$ with dimensions $h_{+}^{p, q}$ and $h_{-}^{p, q}$. Of interest to us will be the third de-Rham cohomology group $H^{3}(\mathcal{X})=H_{+}^{3}(\mathcal{X}) \oplus H_{-}^{3}(\mathcal{X})$, for which we choose a symplectic basis in the following way

$$
\begin{array}{rlrl}
\int_{\mathcal{X}} \alpha_{I} \wedge \beta^{J}=\delta_{I}{ }^{J}, & I, J & =0, \ldots, h_{-}^{2,1}, \\
\left\{\alpha_{I}, \alpha_{a}, \beta^{I}, \beta^{a}\right\}, & \int_{\mathcal{X}} \alpha_{a} \wedge \beta^{b}=\delta_{a}{ }^{b}, & a, b & =1, \ldots, h_{+}^{2,1},
\end{array}
$$

with all other pairings vanishing. A corresponding basis of the third homology group $H_{3}(\mathcal{X})=H_{3+}(\mathcal{X}) \oplus H_{3-}(\mathcal{X})$ together with the non-trivial pairings takes the form

$$
\begin{array}{rlrl}
\int_{A^{I}} \alpha_{J} & =\delta^{I}{ }_{J}, & & \int_{B_{I}} \beta^{J}=\delta_{I}{ }^{J}, \\
\left.\int_{A^{a}} \alpha_{b}, B_{I}, B_{a}\right\}, & \delta^{a}{ }_{b}, & \int_{B_{a}} \beta^{b}=\delta_{a}{ }^{b} .
\end{array}
$$

Special geometry. Next, we consider the special geometry of the complex-structure moduli space. The holomorphic three-form $\Omega$ can be expanded in the symplectic basis $(2.2)$ as follows

$$
\Omega=X^{I} \alpha_{I}-F_{I} \beta^{I}
$$


Since $\Omega$ is odd under the orientifold projection - cf. equation (2.1) - the coefficients of $\Omega$ in the even basis $\left\{\alpha_{a}, \beta^{a}\right\}$ are vanishing, that is $X^{a}=0$ and $\left.F_{a}\right|_{X^{a}=0}=0$. We will refer to these conditions also as the orientifold locus and discuss them in more detail below. The complex-structure moduli are coordinates on a projective space and are usually defined as

$$
z^{i}=\frac{X^{i}}{X^{0}}, \quad z^{i}=u^{i}+i v^{i}, \quad i=1, \ldots, h_{-}^{2,1},
$$

and we note that in our conventions the large-complex-structure limit corresponds to $v^{i} \rightarrow \infty$. For Calabi-Yau compactifications the periods $F_{I}$ can be defined in terms of a prepotential $F$ which is a holomorphic function of degree two in the fields $X^{1}=\left(X^{I}, X^{a}\right)$. More concretely, the $F_{I}$ are defined as the derivatives

$$
F_{I}=\left.\frac{\partial F}{\partial X^{I}}\right|_{X^{a}=F_{a}=0},
$$

which in our present setting have to be evaluated at the orientifold locus. The prepotential $F$ of the $\mathcal{N}=2$ theory consists of a tree-level part involving the triple intersection numbers $d_{\mathrm{ijk}}$ of the mirror Calabi-Yau manifold and one-loop and non-perturbative corrections. More concretely, we have $[11,12]$

$$
\begin{aligned}
F= & \frac{d_{\mathrm{ijk}} X^{\mathrm{i}} X^{\mathrm{j}} X^{\mathrm{k}}}{X^{0}} \\
& +\frac{1}{2} a_{\mathrm{ij}} X^{\mathrm{i}} X^{\mathrm{j}}+b_{\mathrm{i}} X^{\mathrm{i}} X^{0}+\frac{1}{2} c\left(X^{0}\right)^{2}+i\left(X^{0}\right)^{2} \sum_{k} n_{k} \operatorname{Li}_{3}\left(e^{2 \pi i k_{\mathrm{i}} X^{\mathrm{i}} / X^{0}}\right),
\end{aligned}
$$

where the indices $\mathrm{i}, \mathrm{j}, \mathrm{k}=1, \ldots, h^{2,1}$ extend over the even and odd cohomology, $a_{\mathrm{ij}}$ and $b_{\mathrm{i}}$ are rational real numbers whereas $c$ is purely imaginary. ${ }^{1}$ The triple intersection numbers $d_{\mathrm{ijk}}$ and the $a_{\mathrm{ij}}$ are symmetric in their indices, $n_{k}$ are the genus zero Gopakumar-Vafa invariants, $\mathrm{Li}_{3}$ denotes the third poly-logarithm and $k_{\mathrm{i}}$ are the wrapping numbers of worldsheet instantons along two-cycles of the mirror Calabi-Yau.

Orientifold locus. From the $\mathcal{N}=2$ perspective the orientifold locus is determined by $X^{a}=0, F_{a}=0$ for all $a=1, \ldots, h_{+}^{2,1}$. The condition $X^{a}$ alone describes a conifold singularity in which an $A$-cycle shrinks to zero size, and the physics of conifold singularities is well understood [13]. However, imposing in addition $F_{a}=0$ means that also the dual $B$-cycle vanishes. We are not aware of work in the literature studying such situations and we continue with a heuristic discussion.

Let us then study in more detail the condition $F_{a}=0$ evaluated at $X^{a}=0$. From the explicit form of (2.7) we determine

$$
F_{a}=X^{0}\left[3 d_{a i j} z^{i} z^{j}+a_{a i} z^{i}+b_{a}-2 \pi \sum_{k} k_{a} n_{k} \operatorname{Li}_{2}\left(e^{2 \pi i k_{i} z^{i}}\right)\right],
$$

where we recall that $i, j=1, \ldots, h_{-}^{2,1}$ and $a=1, \ldots, h_{+}^{2,1}$. Requiring $F_{a}=0$ for all $z^{i}$ can be solved in the following two ways:

\footnotetext{
${ }^{1}$ These numbers encode the one-loop corrections. We note that there is an ambiguity related to them: for certain non-singular cases they have been computed in [12] but to our knowledge these are not known for singular configurations.
} 
1. A sufficient set of conditions for the periods $F_{a}$ to vanish for all $z^{i}$ reads

$$
d_{a i j}=0, \quad a_{a i}=0, \quad b_{a}=0, \quad k_{a}=0 .
$$

However, especially the condition $k_{a}=0$ is rather restrictive since it means that all world-sheet instanton corrections in the orientifold-even sector have to be absent. This in turn implies a discrepancy in our discussion of emergence in section 4.3.

2. A second possibility is to require a splitting of the sum over $k$ in (2.8) into purely odd and purely even vectors as $\sum_{k}=\sum_{k_{i}}+\sum_{k_{a}}$. Using then the relation $\operatorname{Li}_{2}(1)=\pi^{2} / 6$ we find the conditions

$$
d_{a i j}=0, \quad a_{a i}=0, \quad b_{a}=\frac{\pi^{3}}{3} \sum_{k_{a}} k_{a} n_{k} .
$$

It is not clear to us whether the last of these conditions can be satisfied, but we mentioned in footnote 1 that there is an ambiguity for the one-loop coefficients. The solution (2.10) however realizes $F_{a}=0$ with $k_{a} \neq 0$ which is needed for our discussion in section 4.3 .

\subsection{Effective action}

We now turn to the effective four-dimensional action obtained after compactifying type IIB string theory on the Calabi-Yau orientifolds described above. The details of this action can be found in the literature, and here we focus on the sector corresponding to the third cohomology of $\mathcal{X}$.

Moduli. We compactify type IIB string theory on a Calabi-Yau three-fold as $\mathbb{R}^{3,1} \times \mathcal{X}$ and perform an orientifold projection. The orientifold projection is of the form $\Omega_{\mathrm{P}}(-1)^{F_{\mathrm{L}}} \sigma$, where $\Omega_{\mathrm{P}}$ denotes the world-sheet parity operator and $F_{\mathrm{L}}$ is the left-moving fermion number. The holomorphic involution $\sigma$ acts on $\mathcal{X}$ as in (2.1) and leaves the non-compact four-dimensional part invariant. Hence, the fixed loci of $\sigma$ correspond to orientifold threeand seven-planes. The combination $\Omega_{\mathrm{P}}(-1)^{F_{\mathrm{L}}}$ acts on the massless bosonic fields in the following way

$$
\begin{array}{ll}
\Omega_{\mathrm{P}}(-1)^{F_{\mathrm{L}}} g=+g, & \Omega_{\mathrm{P}}(-1)^{F_{\mathrm{L}}} B=-B, \\
\Omega_{\mathrm{P}}(-1)^{F_{\mathrm{L}}} \phi=+\phi, & \Omega_{\mathrm{P}}(-1)^{F_{\mathrm{L}}} C_{2 p}=(-1)^{p} C_{2 p},
\end{array}
$$

with $g$ the metric, $B$ the Kalb-Ramond field, $\phi$ the dilaton and $C_{2 p}$ the Ramond-Ramond (R-R) potentials in type IIB. The degrees of freedom contained in these fields become fields in the compactified theory, in particular, the deformations of the Calabi-Yau metric are contained in the Kähler form $J$ and the holomorphic three-form $\Omega$. For the RamondRamond four-form potential we note that $C_{4}$ can be expanded as

$$
\begin{aligned}
C_{4}=C_{4}^{(0,4)}+ & C_{4}^{(1,3)}+C_{4}^{(2,2)}+C_{4}^{(4,0)}, \\
\downarrow & \\
& C_{4}^{(1,3)}=U^{a} \wedge \alpha_{a}+V_{a} \wedge \beta^{a},
\end{aligned}
$$


where in $C_{4}^{(m, n)}$ the superscript $m$ denotes the degree in four dimensions and $n$ the degree on the compact space $\mathcal{X}$. Note furthermore that $U^{a}$ and $V_{a}$ correspond to $\mathrm{U}(1)$ vector fields in four dimensions. The four-form $C_{4}^{(4,0)}$ in $\mathbb{R}^{3,1}$ is not dynamical, and due to the self-duality conditions imposed on $C_{4}$ half of the degrees of freedom in the expansion (2.12) are removed. Here we choose to remove $C_{4}^{(2,2)}$ and $V_{a}$ but keep $C_{4}^{(0,4)}$ and $U^{a}$, respectively. The four-dimensional massless fields (in addition to the four-dimensional metric $g_{\mu \nu}$ ) then take the following schematic form

$$
\begin{array}{lll}
\text { complex-structure moduli } & \Omega & \longleftrightarrow H_{-}^{3}(\mathcal{X}), \\
\text { U(1) vector fields } & C_{4}^{(1,3)} & \longleftrightarrow H_{+}^{3}(\mathcal{X}), \\
\text { Kähler moduli } & J+C_{4}^{(0,4)} \longleftrightarrow H_{+}^{2,2}(\mathcal{X}), \\
\text { odd moduli } & B+C_{2} \longleftrightarrow H_{-}^{1,1}(\mathcal{X}), \\
\text { axio-dilaton } & \phi+C_{0} & \longleftrightarrow H_{+}^{0,0}(\mathcal{X}) .
\end{array}
$$

Four-dimensional action. Compactifications of type II string theory on Calabi-Yau manifolds lead to a $\mathcal{N}=2$ supergravity theory in four dimensions, which can be reduced to $\mathcal{N}=1$ by an orientifold projection. Here we are interested in the action corresponding to the complex-structure moduli $z^{i}$ and the vector fields $U^{a}$, which can be brought into the following general form

$$
\mathcal{S} \supset \frac{1}{2} \int_{\mathbb{R}^{3,1}}\left[-\operatorname{Re}\left(f_{a b}\right) F^{a} \wedge \star F^{b}+\operatorname{Im}\left(f_{a b}\right) F^{a} \wedge F^{b}-2 G_{i \bar{j}} d z^{i} \wedge \star d \bar{z}^{\bar{j}}\right] .
$$

The Kähler metric $G_{i \bar{j}}$ for the complex-structure moduli $z^{i}$ appearing in the action (2.14) is computed from a Kähler potential $K_{\mathrm{cs}}$ as

$$
G_{i \bar{j}}=\left.\frac{\partial^{2} K_{\mathrm{cs}}}{\partial z^{i} \partial \overline{\bar{j}} \bar{j}}\right|_{X^{c}=F_{c}=0}, \quad \quad K_{\mathrm{cs}}=-\log \left[-i \int_{\mathcal{X}} \Omega \wedge \bar{\Omega}\right]
$$

and the $\mathrm{U}(1)$ field strength corresponding to $U^{a}$ is denoted by $F^{a}=d U^{a}$. The gauge kinetic function $f_{a b}$ can be expressed in terms of the period matrix $\mathcal{N}$ as

$$
\begin{aligned}
f_{a b} & =-i \overline{\mathcal{N}}_{a b}, \\
\mathcal{N}_{a b} & =\bar{F}_{a b}+2 i \frac{\operatorname{Im}\left(F_{a I}\right) X^{I} \operatorname{Im}\left(F_{b J}\right) X^{J}}{X^{M} \operatorname{Im}\left(F_{M N}\right) X^{N}}=\mathcal{R}_{a b}+i \mathcal{I}_{a b},
\end{aligned}
$$

which follows from evaluating the $\mathcal{N}=2$ gauge kinetic function on the orientifold locus $X^{a}=0, F_{a}=0$ [8]. The period matrix in (2.16) is written using second derivatives of the prepotential $F$, that is

$$
F_{\mathrm{IJ}}=\left.\frac{\partial^{2} F}{\partial X^{\mathrm{I} \partial X^{\mathrm{J}}}}\right|_{X^{c}=F_{c}=0}, \quad \mathrm{I}, \mathrm{J}=0, \ldots, h^{2,1} .
$$

Some expressions. We finally collect some technical results. We first define the inverse of the real part of the gauge kinetic function as the $\mathcal{N}=2$ expression restricted to the orientifold locus, and we obtain using (2.17) that

$$
\left[(\operatorname{Re} f)^{-1}\right]^{a b}=-\left[(\operatorname{Im} F)^{-1}\right]^{a b} .
$$


Second, for the $\mathcal{N}=2$ setting one usually defines a $\left(2 h^{2,1}+2\right) \times\left(2 h^{2,1}+2\right)$ dimensional matrix $\mathcal{M}$ which corresponds to a metric for the third cohomology. We are interested in the orientifold-even part evaluated on the orientifold locus which takes the form

$$
\mathcal{M}=\left(\begin{array}{cc}
-\left[(\operatorname{Im} \mathcal{N})^{-1}\right]^{a b} & {\left[(\operatorname{Im} \mathcal{N})^{-1}(\operatorname{Re} \mathcal{N})\right]_{b}^{a}} \\
{\left[(\operatorname{Re} \mathcal{N})(\operatorname{Im} \mathcal{N})^{-1}\right]_{a}^{b}-\left[(\operatorname{Im} \mathcal{N})+(\operatorname{Re} \mathcal{N})(\operatorname{Im} \mathcal{N})^{-1}(\operatorname{Re} \mathcal{N})\right]_{a b}}
\end{array}\right)
$$

with $a, b=1, \ldots, h_{+}^{2,1}$. Note that this matrix is positive definite. The separate blocks of (2.19) can be determined in terms of (2.17) and take the following form

$$
\begin{aligned}
{\left[(\operatorname{Im} \mathcal{N})^{-1}\right]^{a b} } & =-\left[(\operatorname{Im} F)^{-1}\right]^{a b}, \\
{\left[(\operatorname{Re} \mathcal{N})(\operatorname{Im} \mathcal{N})^{-1}\right]_{a}^{b} } & =-\left[(\operatorname{Re} F)(\operatorname{Im} F)^{-1}\right]_{a}^{b}, \\
{\left[(\operatorname{Im} \mathcal{N})+(\operatorname{Re} \mathcal{N})(\operatorname{Im} \mathcal{N})^{-1}(\operatorname{Re} \mathcal{N})\right]_{a b} } & =-\left[(\operatorname{Im} F)+(\operatorname{Re} F)(\operatorname{Im} F)^{-1}(\operatorname{Re} F)\right]_{a b} .
\end{aligned}
$$

\section{D3-branes wrapping three-cycles}

The main objects of interest for our analysis are D3-branes wrapping three-cycles in the compact geometry, which give rise to particles in the four-dimensional theory.

\subsection{D-branes}

For our purpose we are looking for objects which are charged under the $\mathrm{U}(1)$ gauge fields $U^{a}$. Since the $U^{a}$ are contained in the expansion (2.12) of the R-R four-form $C_{4}$, we are focussing on D-branes.

D3-branes. We first want to show that for the setting introduced above, the only objects coupling directly to the closed-string gauge fields are D3-branes wrapping three-cycles in the compact space. ${ }^{2}$ To do so, we recall the Chern-Simons part of the D-brane action. With $\mu_{p}$ its charge, $\Gamma$ the submanifold wrapped by the D-brane, $\mathcal{F}$ the open-string gauge invariant field strength and the $\hat{\mathcal{A}}$-genus of the curvature two-form, we have

$$
\mathcal{S}_{\mathrm{D} p} \supset-\mu_{p} \int_{\Gamma} \operatorname{ch}(\mathcal{F}) \wedge \sqrt{\frac{\hat{\mathcal{A}}\left(\mathcal{R}_{T}\right)}{\hat{\mathcal{A}}\left(\mathcal{R}_{N}\right)}} \wedge \bigoplus_{q} C_{q} .
$$

Since $\operatorname{ch}(\mathcal{F})$ as well as the $\hat{\mathcal{A}}$-terms are even forms, and since on a Calabi-Yau manifold the first and fifth cohomology are trivial, the only possible D-branes which couple to $C^{(1,3)}$ (cf. the expansion in (2.12)) have to wrap (orientifold-even) three-cycles in $\mathcal{X}$. Furthermore, since $\mathcal{F}$ is odd under $\Omega_{\mathrm{P}}(-1)^{F_{\mathrm{L}}}$ the four-dimensional part does not contain $\mathcal{F}$ and we arrive at a D3-brane wrapping a three-cycle in the compact space $\mathcal{X}$. That means, we consider D3-branes extending along the time direction in the non-compact four-dimensional space $\mathbb{R}^{3,1}$ and wrapping three-cycles $\Gamma_{3} \subset \mathcal{X}$, where the latter can be expanded in the basis $(2.3)$ as follows

$$
\Gamma_{3}=m_{I} A^{I}+m_{a} A^{a}+n^{I} B_{I}+n^{a} B_{a} .
$$

\footnotetext{
${ }^{2}$ As discussed for instance in $[14,15]$, the closed-string $U(1)$ gauge fields can also couple to open-string moduli. This situation is however not generic and will therefore be ignored in the following.
} 
Supersymmetry. The orientifold three- and seven-planes typically present in the background preserve a particular combination of the $\mathcal{N}=2$ supercharges in the four-dimensional theory, leading to $\mathcal{N}=1$. However, D3-branes wrapping three-cycles in the compact space break supersymmetry further. Using the $\kappa$-symmetry formalism for D-branes and following the analysis of [16-19], we find the following:

- A D3-brane wrapping an orientifold-even three-cycle $\Gamma_{3} \in H_{3+}(\mathcal{X})$ breaks supersymmetry completely. The volumes of such three-cycles are expected to vanish, in agreement with the classification of toroidal orientifolds in [20] where the $h_{+}^{2,1}$ sector belongs to the twisted sector. Alternatively, this conclusion is reached by projecting the $\mathcal{N}=2$ expression to $\mathcal{N}=1$.

- A D3-brane wrapping an orientifold-odd three-cycle $\Gamma_{3} \in H_{3-}(\mathcal{X})$ preserves at most one-half of the $\mathcal{N}=1$ supersymmetry in four dimensions, that is, such a D3-brane preserves at most two real supercharges. To do so, a calibration condition of the following form has to be satisfied

$$
\left.\operatorname{Re}\left(e^{i \theta} \Omega\right)\right|_{\Gamma_{3}}=e^{-K_{\mathrm{cs}} / 2} d \operatorname{vol}\left(\Gamma_{3}\right),\left.\quad \operatorname{Im}\left(e^{i \theta} \Omega\right)\right|_{\Gamma_{3}}=0,
$$

where $\theta \in \mathbb{R}$ is an arbitrary phase. It has to be equal for all such D3-branes present in the background. ${ }^{3}$

The important point is that D3-branes wrapping three-cycles in the Calabi-Yau orientifold do not preserve the same supersymmetry as the orientifold three- and seven-planes. In particular, they are not BPS and hence the usual stability arguments do not apply - in general such states are therefore unstable. We come back to this point in section 3.2 below.

Our findings above are consistent with the projection of the $\mathcal{N}=2$ BPS condition for Calabi-Yau compactifications. More concretely, D3-branes wrapping three-cycles in Calabi-Yau three-folds are known to satisfy the inequality [21]

$$
\operatorname{Vol}\left(\Gamma_{3}\right) \geq e^{K_{\mathrm{cs}} / 2}\left|\int_{\Gamma_{3}} \Omega\right|
$$

where the equality corresponds to BPS states in the $\mathcal{N}=2$ theory. When projecting this condition to $\mathcal{N}=1$ via the orientifold projection, we expect corrections to the relation (3.4). However, our expectancy is that these are under control in the large complex-structure regime. Furthermore, since these D3-branes wrap topologically non-trivial cycles in the Calabi-Yau orientifold while being non-BPS, the backreaction on the geometry should be taken into account.

Four-dimensional particles. D3-branes wrapping three-cycles in the compact space can be interpreted as particles in the four-dimensional non-compact theory. The DBI-part of a D-brane action with $\mathcal{F}=0$ has the form $\mathcal{S}_{\mathrm{D} p} \supset-T_{p} \int_{\Gamma} e^{-\phi} d$ vol, where $T_{p}$ denotes

\footnotetext{
${ }^{3}$ For space-time filling D-branes the angle $\theta$ is typically fixed by comparing with corresponding orientifold planes, but such a mechanism is not available here.
} 
the tension of the brane. Going then to Einstein frame we find the following action for D3-branes wrapping $\Gamma_{3}$

$$
\begin{aligned}
\mathcal{S}_{\mathrm{D} 3} & =-T_{3} \int_{\mathbb{R} \times \Gamma_{3}} d s \wedge \operatorname{dvol}\left(\Gamma_{3}\right)-\mu_{3} \int_{\mathbb{R} \times \Gamma_{3}} C_{4} \\
& \sim-m \int_{\mathbb{R}} d s-\int_{\mathbb{R}}\left(m_{a} U^{a}+n^{a} V_{a}\right),
\end{aligned}
$$

where $d s$ is the line element of the world-line in four dimensions. We also included electric as well as magnetic couplings of the D3-brane to the gauge fields and we combine the corresponding charges into the vector $\mathfrak{q}=\left(m_{a}, n^{a}\right)$. Depending on whether the D3-brane wraps an orientifold-even or an orientifold-odd three-cycle, we find the following two classes of particles:

- D3-branes wrapping odd three-cycles $\Gamma_{3} \in H_{3-}(\mathcal{X})$ give rise to massive particles in four dimensions. These are not charged under the $\mathrm{U}(1)$ gauge symmetries and therefore are in general unstable. Using (3.5) and the equality in (3.4) we can then read-off the mass (in units $\sqrt{8 \pi} M_{\mathrm{Pl}}$ ) and charges of these particles as

$$
m=e^{K_{\mathrm{cs}} / 2}\left|m_{I} X^{I}-n^{I} F_{I}\right|+\ldots, \quad \mathfrak{q}=0,
$$

where ... correspond to the aforementioned corrections in the orientifold setting which we expect are subleading in the large complex-structure limit.

- D3-branes wrapping even three-cycles $\Gamma_{3} \in H_{3+}(\mathcal{X})$ give rise to massless fourdimensional particles. This can be explained by noting that explicit constructions of orientifold-even three-cycles in type IIB orientifolds belong to the twisted sector and have vanishing volume [20], or alternatively by projecting the $\mathcal{N}=2$ result. However, these massless particles are charged under the U(1) gauge symmetries with electric charge $m_{a}$ and magnetic charge $n^{a}$, that is

$$
m=0, \quad \mathfrak{q}=\left(\begin{array}{c}
m_{a} \\
n^{a}
\end{array}\right)
$$

D3-branes wrapping a general three-cycle can of course be massive as well as charged, but in these cases the $\mathrm{U}(1)$ charges and their masses are not related to each other.

\subsection{Stability}

Above we have argued that D3-branes wrapping three-cycles in the compact space do not preserve the same supersymmetry as the orientifold three- and seven-planes. They are therefore not BPS states of the four-dimensional theory and thus in general unstable.

D3-branes wrapping orientifold-odd three-cycles. Let us start by briefly recalling the $\mathcal{N}=2$ situation. In this case, D3-branes wrapping special-Lagrangian three-cycles in the compact space give rise to BPS states of the four-dimensional theory. Such states feel an equilibrium of repulsive (gauge interactions) and attractive (gravitational and scalar 
interactions) forces, which allows them to be stable. Furthermore, there can be infinite towers of BPS states as they do not feel a force among themselves. Nevertheless, in order to avoid decay by marginal stability the authors of [7] use a monodromy-orbit formalism to find appropriate bound states building these towers.

In the $\mathcal{N}=1$ orientifold setting, as discussed above, the gauge vectors associated to orientifold-odd three-cycles $\Gamma_{3} \in H_{3-}(\mathcal{X})$ are projected out. The D3-branes wrapping such cycles are massive but uncharged, and therefore feel an attractive potential which usually manifests itself via a tachyonic mode and subsequent tachyon condensation and decay [22, 23]. This is in resemblance to the D-brane of the bosonic string [22, 23], but in our case the spatial extension of the brane is wrapping three-cycles of the Calabi-Yau manifold. As a consequence, without a proper analysis taking into account the backreaction onto the geometry the final state is unknown. The same reasoning applies to towers of such states which are again unstable, reflecting the fact that the monodromy-orbit formalism of [7] is not applicable to these uncharged D3-particles. To summarize, D3-branes wrapping orientifoldodd three-cycles of the Calabi-Yau are in general unstable. We make the following remarks:

- Around equation (3.3) we discussed that some D3-brane configurations preserve $\mathcal{N}=$ $1 / 2$ supersymmetry in four dimensions. These states might have some notion of stability, but a proper analysis is beyond the scope of this work.

- We are not considering non-BPS stability by means of K-theoretical discrete symmetries, since states which are K-theoretical stable are not candidates to build towers of states. In particular, the open-string tachyon for stable non-BPS branes on orientifolds is projected out by world-sheet parity which can only happen for a single brane, whereas towers of more than one brane are unstable and decay [22].

As we have argued, at a generic point in complex-structure moduli space D3-branes wrapping cycles $\Gamma_{3} \in H_{3-}(\mathcal{X})$ are unstable. However, when approaching the large complexstructure limit their mass and their scalar interactions approach zero and these states become "asymptotically stable". In particular, the imbalance of forces gets reduced and backreaction effects become less and less relevant. A motivation for this interpretation is the analysis of emergence for the orientifold-odd sector in section 4.3.

D3-branes wrapping orientifold-even three-cycles. D3-branes wrapping orientifold-even three-cycles $\Gamma_{3} \in H_{3+}(\mathcal{X})$ in the compact space correspond to masslesscharged particles in the four-dimensional theory, which in the context of the swampland program have been discussed for instance in [24, 25]. Many of these particles are however unstable and can decay into their elementary constituents, since there is no mass associated to them. The stable states correspond to the following fundamental charges (for a fixed $a$ ):

$$
\begin{array}{ll}
\left(m_{a}, n^{a}\right)=(1,0) & \text { massless electric particles, } \\
\left(m_{a}, n^{a}\right)=(0,1) & \text { massless magnetic monopoles. }
\end{array}
$$

This reasoning is supported from the $\mathcal{N}=2$ perspective where it was argued in [13] that the conifold singularity is resolved by the addition of the electric state $\left(m_{a}, n^{a}\right)=(1,0)$, 
while the states $\left(m_{a}, n^{a}\right)=(\mathbb{Z}, 0)$ should decay to the previous one in order to match the one-loop beta function for the electric gauge coupling. Since the orientifold locus $X^{a}=0$ resembles a conifold singularity, it is not surprising that we inherit these stable states in the orientifold theory.

Remark. We close this section be remarking that D3-branes wrapping general threecycles can of course be massive as well as charged, but in these cases the U(1) charges and their masses are not related to each other. As a consequence, they ultimately decay to the previous cases.

\section{Swampland conjectures}

We now want to test several swampland conjectures for particles in the setting introduced above. These are the weak gravity conjecture, the swampland distance conjecture and the emergence proposal.

\subsection{Weak gravity conjecture}

We begin our discussion of swampland conjectures with the weak gravity conjecture.

Recalling the conjectures. We start by briefly recalling the electric weak gravity conjecture [3] in its modern formulation [2]:

Consider a U(1) gauge theory with gauge coupling $g$ coupled to gravity and described by the following action

$$
\mathcal{S}=\frac{1}{2} \int_{\mathbb{R}^{3,1}}\left[M_{\mathrm{P}}^{2} R \star 1-\frac{1}{2 g^{2}} F \wedge \star F+\ldots\right],
$$

where $R$ denotes the Ricci scalar and $F$ is the $\mathrm{U}(1)$ field-strength two-form. Then there exists a particle in the theory with mass $m$ and charge $\mathfrak{q}$ satisfying the inequality

$$
m \leq \sqrt{2} \mathfrak{q} g M_{\mathrm{P}} .
$$

This conjecture has been refined in many ways, and for our purpose the weak gravity conjecture with scalar fields will be relevant [4]. We recall this conjecture as follows:

Consider a gravity theory with massless scalar fields $z^{i}$ and $\mathrm{U}(1)$ gauge fields $U^{a}$ described by the action (2.14). Then there should exist a particle with mass $m(z)$ satisfying the bound

$$
\mathcal{Q}^{2} \geq m^{2}+G^{i \bar{j}}\left(\partial_{z^{i}} m\right)\left(\partial_{\bar{z}^{j}} m\right), \quad \mathcal{Q}^{2}=\frac{1}{2} \mathcal{Q}^{T} \mathcal{M} \mathcal{Q},
$$

where $\mathcal{Q}$ denotes the vector $\mathcal{Q}=\left(m_{I}, m_{a}, n^{I}, n^{a}\right), \mathcal{M}$ is the positive-definite matrix similar to the one defined in (2.19) and $G^{i \bar{j}}$ is the inverse of the Kähler metric (2.15). 
Verifying the conjectures. We now discuss how these conjectures are satisfied in the orientifold setting introduced in the previous section:

- For D3-branes wrapping odd three-cycles $\Gamma_{3} \in H_{3-}(\mathcal{X})$ we recall from (3.6) that their $\mathrm{U}(1)$ charges $\mathfrak{q}=\left(m_{a}, n^{a}\right)$ are vanishing. These particles do not provide the required charged particle for the weak gravity conjecture (4.2), but one can check that they verify the scalar weak gravity conjecture (4.3) without U(1) gauge interactions [4].

- D3-branes wrapping even three-cycles $\Gamma_{3} \in H_{3+}(\mathcal{X})$ are massless and their charges $\mathfrak{q}$ are given by the wrapping numbers $\left(m_{a}, n^{a}\right)$. The electric weak gravity conjecture (4.2) is therefore verified. Furthermore, using (3.7) we find that

$$
\frac{1}{2}\left(\begin{array}{c}
m \\
n
\end{array}\right)^{T} \mathcal{M}\left(\begin{array}{c}
m \\
n
\end{array}\right) \geq 0
$$

which is satisfied since the matrix $\mathcal{M}$ is positive definite.

We therefore conclude that the electric weak gravity conjecture (4.1) and weak gravity conjecture with scalar fields (4.3) are trivially satisfied.

Completeness conjecture and tower versions. The statement of the completeness conjecture is that a gravity theory with a gauge symmetry must have states of all possible charges under that gauge symmetry [26]. In our case this conjecture is satisfied by D3branes wrapping orientifold-even cycles $\Gamma_{3} \in H_{3+}(\mathcal{X})$ with wrapping numbers $\left(m_{a}, n^{a}\right)$. However, in general these states are not stable against decay to the elementary ones (3.8) and hence the completeness conjecture is only satisfied as long as stability is not required. The completeness conjecture motivates the tower versions of the weak gravity conjecture [27-29], which suggests that for the D3-brane particles discussed here the tower versions are satisfied only if stability of the states is relaxed.

Furthermore, in [2] it is argued that avoidance of generalized global symmetries for an $\mathrm{U}(1)$ gauge theory coupled to gravity requires at least one single charged state. Remarkably, in [30] it is claimed that an extension of the previous argument to discrete generalized global symmetries requires all possible charges in order to break any possible discrete symmetry. It would be interesting to explore the compatibility between our results concerning the completeness conjecture and [30].

Summary and remark. We now want to briefly summarize our findings regarding the weak gravity conjecture and make the following remark:

- We have shown that the weak gravity conjectures for particles are verified in type IIB orientifold compactifications with O3- and O7-planes for D3-branes wrapping three-cycles. However, the tower versions cannot be claimed to be verified as long as stability is required.

- Analogously to type IIB orientifolds with O3-/O7-planes case studied here, the same results are obtained for type IIB orientifolds with O5-/O9-planes and for type IIA orientifolds. 
- In [9] it was shown that in the orientifold setting BPS D5-branes wrapping threecycles verify the weak gravity conjecture as well. These are however extended objects and not particles, and they are charged under a different gauge symmetry.

\subsection{Swampland distance conjecture}

We next consider the swampland distance conjecture for type IIB orientifolds with O3/O7-planes introduced above.

Recalling the conjecture. The swampland distance conjecture has been formulated in [5], and has been refined in [6]. The refined version reads as follows:

Consider a theory coupled to gravity with a moduli space $\mathcal{M}_{\text {mod }}$ parametrized by the expectation values of some fields without potential. Let the geodesic distance between any two points $P, Q \in \mathcal{M}_{\text {mod }}$ be denoted $d(P, Q)$. If $d(P, Q) \gtrsim M_{\mathrm{P}}$, then there exists an infinite tower of states with mass scale $m$ such that

$$
m(Q)<m(P) e^{-\mu \frac{d(P, Q)}{M_{\mathrm{P}}}},
$$

where $\mu$ is some positive constant of order one. This statement holds even for fields with a potential, where the moduli space is replaced with the field space in the effective theory.

We now want to verify this conjecture (in the large complex-structure domain) for the setting of the previous section. In particular, we start from a generic large complexstructure point and approach another large complex-structure point along a geodesic in moduli space. ${ }^{4}$ The results obtained in [7] for the $\mathcal{N}=2$ theory suggest that also in our $\mathcal{N}=$ 1 orientifold setting D3-branes wrapping three-cycles give rise to the infinite tower of states.

- We have argued that the mass of D3-branes wrapping even three-cycles $\Gamma_{3} \in H_{3+}(\mathcal{X})$ vanishes and hence they do not play a role for the swampland distance conjecture.

- For D3-branes wrapping odd three-cycles $\Gamma_{3} \in H_{3-}(\mathcal{X})$ the $\mathrm{U}(1)$ charges are vanishing but they are massive (cf. equation (3.6)). These states are therefore of interests for the swampland distance conjecture.

Geodesic distance. Let us consider a generic point in complex-structure moduli space for which the one-loop and non-perturbative corrections to the prepotential (2.7) can be ignored. The Kähler metric is computed via (2.15), and the geodesic equation for such Kähler geometries reads in general

$$
0=\ddot{z}^{i}+\Gamma_{j k}^{i} \dot{z}^{j} \dot{z}^{k}, \quad \Gamma_{j k}^{i}=G^{i \bar{m}} \partial_{j} G_{k \bar{m}},
$$

\footnotetext{
${ }^{4}$ Note that our analysis excludes the regions of complex-structure moduli space where the one-loop and non-perturbative corrections to the prepotential (2.7) are relevant. Furthermore, the beginning of the region of large complex structure where the instability corrections, specific of our $\mathcal{N}=1$ setting, can be disregarded is quantitatively unknown. We are therefore testing only a weaker version of the refined swampland distance conjecture, in particular, we do not determine where in moduli space the exponential behaviour of the tower of states starts. For work on the refined swampland distance conjecture including the non-geometric phases of moduli space see for instance [31].
} 
where a dot indicates a derivative with respect to the parametrization $t$ of the geodesic. The geodesic distance is defined as

$$
d(P, Q)=\left|\int_{t_{1}}^{t_{2}} d t \sqrt{2 \dot{z}^{i} G_{i \bar{j}} \dot{\bar{z}} \bar{j}}\right|, \quad \begin{aligned}
& z^{i}\left(t_{1}\right)=P \in \mathcal{M}_{\text {mod }} \\
& z^{i}\left(t_{2}\right)=Q \in \mathcal{M}_{\text {mod }}
\end{aligned}
$$

For simplicity we now restrict ourselves to a situation with one complex-structure modulus, that is $h_{-}^{2,1}=1$. We use the notation $z^{1} \equiv z=u+i v$, and the moduli space is a hyperbolic space with metric

$$
G_{1 \overline{1}}=\frac{3}{4} \frac{1}{v^{2}} .
$$

The solutions to the geodesic equation (4.6) are well known, and correspond to lines with constant $u$ and to circles intersecting $v=0$ at a right angle

$$
z_{(1)}=\alpha+i \beta e^{t}, \quad z_{(2)}=\alpha+\frac{\beta}{\cosh (t)}[\sinh (t)+i]
$$

where $\alpha, \beta=$ const and $\beta>0$. The geodesic distance for these two cases is computed as follows

$$
\begin{aligned}
& d_{(1)}(P, Q)=\sqrt{\frac{3}{2}}\left|t_{Q}-t_{P}\right|=\sqrt{\frac{3}{2}}\left|\log \frac{v_{Q}}{v_{P}}\right|, \\
& d_{(2)}(P, Q)=\sqrt{\frac{3}{2}}\left|t_{Q}-t_{P}\right| .
\end{aligned}
$$

Verifying the conjecture. As mentioned above, motivated by the $\mathcal{N}=2$ results we expect that D3-branes wrapping orientifold-odd three-cycles will provide the tower of states which becomes exponentially light. In our example with $h_{-}^{2,1}=1$ we see that D3-branes with wrapping numbers $n^{0} \neq 0$ or $n^{1} \neq 0$ do not satisfy the swampland distance conjecture (4.5) in the large complex-structure limit $v \rightarrow \infty$; they are expected to provide the required states in the small complex-structure limit. We focus here on the large complexstructure limit and D3-branes with wrapping numbers $m_{0}$ and $m_{1}$ :

- We first consider D3-branes satisfying the calibration condition (3.3). In this case the mass is given by

$$
m(z)=\frac{1}{\sqrt{8 d_{111} v^{3}}} \operatorname{Re}\left[e^{i \hat{\theta}}\left(m_{0}+m_{1} z+n^{0} d_{111} z^{3}-3 n^{1} d_{111} z^{2}\right)\right],
$$

with $d_{111}$ the triple intersection number introduced in (2.7) and where we defined $\hat{\theta}=\theta+\arg \left(X^{0}\right)$. If we approach the large complex-structure limit via the first geodesic in (4.9), it turns out that there are only two choices for $\hat{\theta}$ compatible with the calibration condition. The tower satisfying the swampland distance conjecture together with the constant $\mu$ are the following

$$
\begin{aligned}
& \hat{\theta}=0, \quad m_{0}>0, \quad m_{1}=0, \quad n^{a}=0, \quad \mu=\sqrt{3 / 2}, \\
& \hat{\theta}=\pi / 2, \quad m_{0}=0, \quad m_{1}>0, \quad n^{a}=0, \quad \mu=\sqrt{1 / 6} .
\end{aligned}
$$


- Next, we relax the calibration condition and consider states satisfying the equality in (3.4). These are the states inherited from the $\mathcal{N}=2$ theory, and their mass is given by

$$
m(z)=\frac{1}{\sqrt{8 d_{111} v^{3}}}\left|m_{0}+m_{1} z+n^{0} d_{111} z^{3}-3 n^{1} d_{111} z^{2}\right| .
$$

Approaching the large complex-structure limit again via first geodesic in (4.9), we see that tower of states satisfying the swampland distance conjecture are given by

$$
m_{0} \in \mathbb{Z}, \quad m_{1} \in \mathbb{Z}, \quad n^{a}=0, \quad \mu=\sqrt{1 / 6} .
$$

- We also want to consider the second geodesic in (4.9), for which the large complexstructure limit is characterized by $\beta \ggg 1$ and $t \rightarrow 0$. For this geodesic we do not find any tower of D3-brane states satisfying the swampland distance conjecture. However, since this path is along the boundary of the complex-structure moduli space it is not clear whether the conjecture applies. It would be interesting to study this point further.

To summarize, along the path $u=$ const. and $v \rightarrow \infty$ we have verified the swampland distance conjecture for D3-particles in type IIB orientifold compactifications in the large complex-structure domain, however, the tower of states is in general not stable. Furthermore, we were not able to verify the swampland distance conjecture for a path along the boundary of complex-structure moduli space.

Relation between swampland distance and weak gravity conjectures. For $\mathcal{N}=2$ theories originating from type IIB Calabi-Yau compactifications it was found in [7] that the swampland distance as well as the weak gravity conjecture are verified by BPS D3-branes wrapping special Lagrangian three-cycles. However, for $\mathcal{N}=1$ theories obtained via an orientifold projection we have noted the $h^{2,1}$ vector multiplets in $\mathcal{N}=2$ are projected to $h_{-}^{2,1}$ chiral and $h_{+}^{2,1}$ vector multiplets in $\mathcal{N}=1$ (cf. equation (1.1)). In particular, in the orientifold theory both multiplets are independent of each other, and there exist for instance orientifolds with $h_{+}^{2,1}=0$. Thus, D3-brane particles which are needed for the swampland distance conjecture are uncharged and trivially do not satisfy the electric weak gravity conjecture (4.2) - and charged D3-brane particles satisfy the weak gravity conjecture but are not needed for the swampland distance conjecture. Thus, the relation between the weak gravity and swampland distance conjectures (and emergence to be discussed below) is determined by the bulk supergravity spectrum.

This observation extends to similar cases for which D-branes couple to a bulk spectrum in $\mathcal{N}=2$ which splits under the orientifold projection into different $\mathcal{N}=1$ multiplets. For example, in Kähler moduli space the same happens for D-branes wrapping two-cycles (e.g. the D-strings studied in [9]) coupling to both $J$ and $C_{2}$. It is also worth mentioning that similar to the complex-structure moduli, also the Kähler twisted sector of the examples in [20] contains the $h_{-}^{1,1}$ cycles, so we observe an analogy with respect to the situation studied in this work. 


\subsection{Emergence proposal}

In this section we discuss the emergence proposal for D3-branes wrapping three-cycles in type IIB orientifold compactifications with orientifold three- and seven-planes.

Recalling the proposal. The emergence proposal has been formulated in $[2,7]$, which we recall as follows:

The dynamics (kinetic terms) for all fields are emergent in the infrared by integrating out towers of states down from an ultraviolet scale $\Lambda_{\mathrm{UV}}$ which is below the Planck scale.

At a practical level this means that at an UV scale $\Lambda_{\mathrm{UV}}$, the renormalization group flow has a boundary condition on all fields forcing them to have vanishing kinetic terms. Let us make the following remarks concerning the emergence proposal:

- For towers of states with equidistant mass and charge separation and without taking care of higher-loop corrections, it has been argued in [2] that imposing emergence of gravity one obtains the species scale $\Lambda_{\mathrm{S}}[32]$ as the UV scale.

- Imposing emergence of a gauge field and a scalar field (to one-loop order) one recovers the magnetic weak gravity and the swampland distance conjecture, respectively. In [7] this mechanism has been discussed for type IIB Calabi-Yau compactifications with towers of states given by D3-branes wrapping special Lagrangian three-cycles.

- For orientifold compactifications with $\mathcal{N}=1$ supersymmetry in four dimensions we expect a modified picture. Indeed, the non-renormalization theorem for $\mathcal{N}=$ 2 changes in the case of $\mathcal{N}=1$ orientifolds as the Kähler potential $K_{\text {cs }}$ receives loop corrections in terms of Eisenstein series [33]. These corrections are however subleading and expected to be irrelevant as we approach the large complex-structure limit. Besides, the gauge kinetic functions remain one-loop exact.

Emergence for the orientifold-odd third homology. We now analyze the emergence proposal for the orientifold-odd third cohomology. As discussed above, D3-branes wrapping cycles $\Gamma_{3} \in H_{3-}(\mathcal{X})$ are massive but unstable. Integrating out these states gives rise to logarithmic corrections to the complex-structure moduli-space metric of the schematic form

$$
\left.G_{i \bar{j}}\right|_{\mathrm{IR}}=\left.G_{i \bar{j}}\right|_{\mathrm{UV}}+c_{-} \sum_{\alpha}\left(\partial_{z^{i}} m^{(\alpha)}\right)\left(\partial_{\bar{z}^{j}} m^{(\alpha)}\right) \log \frac{\Lambda_{\mathrm{UV}}}{m^{(\alpha)}},
$$

where $c_{-}$is a normalization constant and the sum runs over all D3-brane states with mass below the cutoff scale $\Lambda_{\mathrm{UV}}$. Following the argumentation of $[2,7]$ for the emergence proposal in the $\mathcal{N}=2$ setting, the metric $\left.G_{i \bar{j}}\right|_{\text {UV }}$ in the UV vanishes while the sum over logarithmic corrections generates the metric $\left.G_{i \bar{j}}\right|_{\mathrm{IR}}$ in the IR. It is beyond the scope of this work to check (4.15) explicitly for our setting, but we would like to make the following comments:

- For the orientifold setting studied in this work we have argued that the tower of D3-brane states is in general unstable. In order to verify the emergence proposal we therefore have to integrate-out (infinite) unstable states, which are however expected to become asymptotically-stable in the large complex-structure limit. 
- In [9] it has been proposed that also four-dimensional domain walls contribute to the running of the moduli-space metric. These domain walls are given by BPS D5-branes wrapping three-cycles in the compact space, but to our knowledge it is not known how to integrate out extended objects at the quantitative level. This ambiguity is partially reflected in the unspecified constant $c_{-}$in (4.15).

- It is curious to note that in the $\mathcal{N}=1$ setting we have in general all-order loop corrections to the Kähler potential but also that the D3-brane particles being integrated out are not stable.

We believe these questions deserve further investigation in the future.

Emergence for the orientifold-even third homology. We now consider D3-branes wrapping orientifold-even three-cycles $\Gamma_{3} \in H_{3+}(\mathcal{X})$ in the compact space. These states are massless but are charged under the closed-string $\mathrm{U}(1)$ gauge fields $U^{a}$, and therefore contribute to one-loop corrections to the gauge kinetic function. In particular, in field theory we have

$$
\left.\operatorname{Re} f_{a b}^{(\mathrm{f})}\right|_{\mathrm{IR}}=\left.\operatorname{Re} f_{a b}^{(\mathrm{f})}\right|_{\mathrm{UV}}+c_{+} \lim _{m^{(\alpha)} \rightarrow 0} \sum_{\alpha} \mathfrak{q}_{a}^{(\alpha)} \mathfrak{q}_{b}^{(\alpha)} \log \frac{\Lambda_{\mathrm{UV}}}{m^{(\alpha)}}
$$

where the sum is over all D3-brane particles labelled by $\alpha, \mathfrak{q}_{a}^{(\alpha)}$ denote their electric charges and $m^{(\alpha)}$ denote their masses which vanish at the orientifold locus. The constant $c_{+}$is a normalization constant, and the superscript indicates that this is a field-theory result. As expected, we obtain logarithmic divergences due to massless particles running in the loop.

Let us now turn to the string-theory computation. Using the relation (2.16) and the explicit form of the prepotential (2.7), we find at the orientifold locus $X^{a}=0$ (without imposing $F_{a}=0$ )

$$
\operatorname{Re} f_{a b}^{(\mathrm{s})}=\operatorname{Im} F_{a b}=6 d_{a b i} v^{i}+(2 \pi)^{2} \sum_{k} k_{a} k_{b} n_{k} \operatorname{Re} \log \left(1-e^{2 \pi i k_{i} z^{i}}\right) .
$$

Here we used again $v^{i}=\operatorname{Im} z^{i}$ and the superscript indicates that this is the (effective) string-theory result. Following now the philosophy of the emergence proposal $[2,7]$, the gauge-kinetic function should vanish in the ultraviolet and the expression in the infrared is generated by loop corrections. In the present situation we argue as follows:

- The gauge-kinetic function in the infrared is given by the expression (4.17). Let us then recall our discussion from page 4 and consider the solution (2.10) to the orientifold condition $F_{a}=0$. Reinstalling the $X^{a}$-dependence we have

$$
\operatorname{Re} f_{a b}^{(\mathrm{s})}=6 d_{a b i} v^{i}+(2 \pi)^{2} \lim _{X^{a} \rightarrow 0} \sum_{k} k_{a} k_{b} n_{k} \operatorname{Re} \log \left(-2 \pi i k_{a} X^{a} / X^{0}\right) .
$$

Recalling furthermore our results for the masses from page 9 and performing a similar analysis for D3-branes wrapping orientifold-even cycles, we find for electricallycharged particles that $m=\lim _{X^{a} \rightarrow 0} e^{K_{\mathrm{cs}} / 2}\left|m_{a} X^{a}\right|$ (in units of $M_{\mathrm{Pl}}$ ) with $m_{a}$ given 
by (3.8). Using this expression in (4.18), we obtain up to prefactors and regular terms

$$
\operatorname{Re} f_{a b}^{(\mathrm{s})} \sim \lim _{m^{(\alpha)} \rightarrow 0} \sum_{\alpha} k_{a}^{(\alpha)} k_{b}^{(\alpha)} \log \frac{M_{\mathrm{s}}}{m^{(\alpha)}},
$$

where $M_{\mathrm{s}}$ denotes the string scale and where we relabeled the vectors $k$ appearing in the sum by $\alpha$. This expression has the same form as the field-theoretical one-loop correction shown in (4.16), and thus it is feasible that the emergence proposal is verified.

- The above-mentioned string-theory result for the gauge-kinetic function is not complete. Indeed, the results of [13] imply that (4.17) receives corrections in the ultraviolet from D3-branes wrapping once around orientifold-even cycles. Properly taking into account these states cure the logarithmic divergence in the Calabi-Yau moduli space, and hence the gauge-kinetic functions (4.17) and (4.18) are expected to be finite in a quantum-gravity theory. In particular, in the infrared we expect from string theory a behavior of the form

$$
\left.\operatorname{Re} f_{a b}^{(\text {full })}\right|_{\mathrm{IR}}=6 d_{a b i} v^{i}+\text { finite, }
$$

with the superscript indicating the full expression.

To summarize, we have argued that the emergence proposal for D3-branes wrapping orientifold-even three-cycles is feasible to be verified, though we have presented here only qualitative analysis and a more thorough check including numerical factors and regular terms would be necessary.

Remarks and open questions. Let us make the following remarks concerning our discussion of emergence in the orientifold-even sector:

- In our analysis we have encountered a difference between the field-theory and the string-theory computation. In particular, the field-theory expression (4.16) for the gauge-kinetic function is logarithmically divergent whereas the full string-theory expression (4.20) is expected to be finite.

- The gauge-kinetic function (4.20) is the expression expected from string theory once the effect of D3-branes wrapping orientifold-even cycles on the moduli-space geometry has been taken into account. For the emergence proposal this gauge coupling in the infrared should be obtained entirely from loop-corrections with charged particles running in the loop, however, in our case these charged particles are massless and do not couple to the complex-structure moduli. It is therefore not clear to us how the $v^{i}$ as well as $d_{a b i}$ dependence in (4.20) can be obtained.

- A potential answer to this question is that there exist particles which couple simultaneously to the $\mathrm{U}(1)$ gauge fields and to the complex-structure moduli, and where the coupling depends on $d_{a b i}$, such that generate the linearly divergent term when taking into account their effect in (4.16). However, there are no other known states with this property. 
- Another possibility is that the triple intersection numbers $d_{a b i}$ vanish. This appears to be a too-strong requirement, since in T-dual settings these can be non-vanishing.

- Since D3-branes wrapping orientifold-even three-cycles do not preserve the bulk supersymmetry (see our discussion on page 8), the gauge-kinetic function may receive higher-loop corrections which can generate a term linear in $v^{i}$.

- We also note that when taking the large complex-structure limit in (4.20), the gauge symmetry becomes global. Although there exists an obstruction against reaching the limit $v^{i} \rightarrow \infty$ due to towers of orientifold-odd D3-branes, it is unexpected to find a global gauge symmetry associated with integrating out states uncharged under it. In particular, this behavior is usually associated with integrating out a tower of states which becomes massless as in (4.5) and which is charged under the gauge symmetry. However, in our setting we do not realize a possible tower of states verifying both properties.

We leave open these questions here and refer for a more detailed description and further instructive attempts to solve them to section 6 of [10].

\section{$5 \quad$ Summary and conclusions}

In this paper we have studied swampland conjectures for type IIB orientifolds with O3- and O7-planes. We have allowed for orientifold projections with $h_{+}^{2,1} \neq 0$, which leads to closedstring U(1) gauge fields in four dimensions. The weak gravity conjecture, the swampland distance conjecture and emergence proposal have been investigated in the context of the $\mathcal{N}=2$ parent theory in [7], and here we were interested in the question What happens to the $\mathcal{N}=2$ analysis when we perform an orientifold projection?

Summary. Let us summarize the main results of our analysis:

- The relevant objects for the above-mentioned swampland conjectures in our setting are D3-branes wrapping three-cycles in the compact space. In the $\mathcal{N}=2$ theory these couple to vector multiplets, and they give rise to four-dimensional particles charged under $\mathrm{U}(1)$ gauge fields and with mass depending on the complex-structure moduli. In the orientifold theory the $\mathcal{N}=2$ vector multiplets split into vector and chiral multiplets of $\mathcal{N}=1$ supergravity as illustrated in (1.1). Correspondingly, D3-branes can be separated into massive-uncharged (orientifold-odd) and masslesscharged (orientifold-even) particles in four dimensions. While for $\mathcal{N}=2$ these states can be BPS and therefore can be stable, for the $\mathcal{N}=1$ theory they are in general unstable and non-supersymmetric. This suggests that the $\mathcal{N}=1$ swampland conjectures studied in this paper are satisfied only for in general unstable states.

- We have illustrated that the weak gravity conjecture [3] and the weak gravity conjecture with scalar fields [4] are trivially satisfied in the $\mathcal{N}=1$ setting. This is true both for D3-branes wrapping orientifold-odd and orientifold-even three-cycles, however, the tower versions can only be satisfied if the stability requirement is relaxed. 
- We have verified the swampland distance conjecture $[5,6]$ (in the large complexstructure domain) for D3-branes wrapping orientifold-odd three-cycles in the CalabiYau three-fold in the case $h_{-}^{2,1}=1$ for the large complex-structure limit. For this setting we studied two types of geodesics, where one geodesic along the boundary of the complex-structure moduli space appears to violate the conjecture. We noted furthermore that the massless-charged D3-brane particles do not contribute to the swampland distance conjecture, and that the tower of states is only asymptotically stable.

- For the emergence proposal $[2,7]$ we have seen that integrating out D3-branes wrapping orientifold-odd three-cycles gives rise to the correct behavior of the Kähler metric for the complex-structure moduli. The analysis in the orientifold-even sector was more ambiguous, but we argued that D3-branes wrapping (collapsing) three-cycles can re-produce the leading divergency in the gauge-kinetic function for the closed-string U(1) gauge fields. However, when properly taking into account the effect of such D3-branes on the moduli-space geometry it is expected that this divergence is removed. There is furthermore an open question of how the leading regular term in the gauge-kinetic function can be obtained from loop corrections, and we briefly discussed possible solutions.

Open questions and future directions. We comment on open questions and future research directions:

- The closed-string gauge theories originating from the orientifold-even sector have been studied mostly from a supergravity point of view and require a better understanding within string theory. More concretely, the implications of the orientifold locus $X^{a}=$ $0, F_{a}=0$ on the world-sheet instanton corrections (cf. page 4) need to be clarified, the properties of D3-branes wrapping three-cycles $\Gamma_{3} \in H_{3+}(\mathcal{X})$ deserved to be studied further, and the emergence proposal for the gauge theories should be made more explicit. To do so a concrete example would be helpful, for which the results in [20] provide a good starting point..$^{5}$

- While in the $\mathcal{N}=2$ setting D3-branes wrapping three-cycles in the compact space can be BPS, this property is lost after orientifolding. More concretely, the corresponding states in the four-dimensional theory no longer preserve supersymmetry and we expect corrections to the mass formula for orientifold-odd D3-branes shown in (3.6), associated with their instability and with backreaction effects onto the geometry. We have argued that these effects should be subleading as we approach the infinite-distance limit and have disregarded them, however, such corrections should be computed explicitly.

This is in line with results obtained in [35], where logarithmic quantum corrections to various swampland conjectures — including the de-Sitter swampland conjecture

\footnotetext{
${ }^{5}$ A certain orientifold of $\mathbb{T}^{6} / \mathbb{Z}_{4}$ with $\left(h_{-}^{2,1}, h_{+}^{2,1}\right)=(0,1)$ has been studied in a different context in section 4.1 of [34]. Due to the absence of complex-structure moduli this example is not suitable for the present purpose, however, the gauge-kinetic function found there agrees with the expectations of our work.
} 
- have been discussed. At the same time, the authors of [36] have connected the de-Sitter conjecture to the swampland distance and weak gravity conjectures. If these connections survive in the orientifold setting, it would be interesting to see if through them the work of [35] can shine light on the concrete form of possible modifications to the mass formula (3.6), to the swampland distance and to weak gravity conjectures for our setting.

- In this work we focused on type IIB orientifolds with O3- and O7-planes. It would be interesting to extend our discussion to type IIA orientifolds where a similar splitting of the $\mathcal{N}=2$ vector multiplet occurs [37]. From mirror symmetry we expect that the mirror towers/D-branes satisfy a similar behavior, which for the $\mathcal{N}=2$ case was shown in [38]. Besides, the E2-instantons analyzed in [39] have properties similar to the case we studied but in the complex-structure moduli space of type IIA. Furthermore, in the work of [9] it would be interesting to explore the role of the uncharged towers of extended objects.

- We have analysed the swampland distance conjecture for complex-structure moduli in the large complex-structure limit for the case $h_{-}^{2,1}=1$. It would be desirable to extend this single-modulus analysis to multi-moduli settings and general moduli-space singularities. For $\mathcal{N}=2$ Calabi-Yau compactifications a classification of infinitedistance networks can be found in [40] and a concrete two-moduli example has been worked-out in [41]. However, for $\mathcal{N}=1$ orientifold compactifications these results would have to be reassessed along the lines of this work.

- Many studies of the swampland conjectures are based on $\mathcal{N} \geq 2$ supergravity theories (see [42] for recent work with extended supersymmetry). However, in order to make contact with phenomenology, theories with $\mathcal{N}=1$ supersymmetry are more suitable. What we find in our work is that many of the connections between the swampland conjectures and the emergence proposal are modified for $\mathcal{N}=1$, and therefore further studies of these connections and their implications for phenomenology would be desirable.

\section{Acknowledgments}

We would like to thank R. Blumenhagen, L. Martucci and E. Palti for very helpful discussions and we thank D. Lüst for support. EP thanks K. Schalm for hospitality and L. Plauschinn for enlightening discussions. MER is grateful to H. Erbin for suggestions on literature concerning tachyon condensation in string field theory [23] and non-BPS stability [22] as well as to H. Ásmundsson, D. Bockisch, P. Fragkos, D.P. Lichtig, A. Makridou, I. Mayer, S.P. Mazloumi and J.D. Simão for patient and useful discussions and finally to his family and friends for unconditional support along the period of elaboration of this work.

Open Access. This article is distributed under the terms of the Creative Commons Attribution License (CC-BY 4.0), which permits any use, distribution and reproduction in any medium, provided the original author(s) and source are credited. 


\section{References}

[1] C. Vafa, The string landscape and the swampland, hep-th/0509212 [INSPIRE].

[2] E. Palti, The swampland: introduction and review, Fortsch. Phys. 67 (2019) 1900037 [arXiv: 1903.06239] [INSPIRE].

[3] N. Arkani-Hamed, L. Motl, A. Nicolis and C. Vafa, The string landscape, black holes and gravity as the weakest force, JHEP 06 (2007) 060 [hep-th/0601001] [INSPIRE].

[4] E. Palti, The weak gravity conjecture and scalar fields, JHEP 08 (2017) 034 [arXiv: 1705. 04328] [INSPIRE].

[5] H. Ooguri and C. Vafa, On the geometry of the string landscape and the swampland, Nucl. Phys. B 766 (2007) 21 [hep-th/0605264] [INSPIRE].

[6] D. Klaewer and E. Palti, Super-Planckian spatial field variations and quantum gravity, JHEP 01 (2017) 088 [arXiv: 1610.00010] [INSPIRE].

[7] T.W. Grimm, E. Palti and I. Valenzuela, Infinite distances in field space and massless towers of states, JHEP 08 (2018) 143 [arXiv:1802.08264] [INSPIRE].

[8] T.W. Grimm and J. Louis, The effective action of $N=1$ Calabi-Yau orientifolds, Nucl. Phys. B 699 (2004) 387 [hep-th/0403067] [INSPIRE].

[9] A. Font, A. Herráez and L.E. Ibáñez, The swampland distance conjecture and towers of tensionless branes, JHEP 08 (2019) 044 [arXiv: 1904.05379] [INSPIRE].

[10] M. Enríquez, Swampland conjectures for $\mathcal{N}=1$ orientifolds, M.Sc. thesis, Arnold Sommerfeld Center, Ludwig Maximilians Universität, Munich, Germany (2019).

[11] S. Hosono, A. Klemm and S. Theisen, Lectures on mirror symmetry, Lect. Notes Phys. 436 (1994) 235 [hep-th/9403096] [INSPIRE].

[12] S. Hosono, A. Klemm, S. Theisen and S.-T. Yau, Mirror symmetry, mirror map and applications to complete intersection Calabi-Yau spaces, AMS/IP Stud. Adv. Math. 1 (1996) 545 [hep-th/9406055] [INSPIRE].

[13] A. Strominger, Massless black holes and conifolds in string theory, Nucl. Phys. B 451 (1995) 96 [hep-th/9504090] [INSPIRE].

[14] H. Jockers, The effective action of D-branes in Calabi-Yau orientifold compactifications, Fortsch. Phys. 53 (2005) 1087 [hep-th/0507042] [INSPIRE].

[15] F. Marchesano, D. Regalado and G. Zoccarato, U(1) mixing and D-brane linear equivalence, JHEP 08 (2014) 157 [arXiv: 1406.2729] [InSPIRE].

[16] E. Bergshoeff and P.K. Townsend, Super D-branes, Nucl. Phys. B 490 (1997) 145 [hep-th/9611173] [INSPIRE].

[17] E. Bergshoeff, R. Kallosh, T. Ortín and G. Papadopoulos, Kappa symmetry, supersymmetry and intersecting branes, Nucl. Phys. B 502 (1997) 149 [hep-th/9705040] [INSPIRE].

[18] M. Mariño, R. Minasian, G.W. Moore and A. Strominger, Nonlinear instantons from supersymmetric p-branes, JHEP 01 (2000) 005 [hep-th/9911206] [INSPIRE].

[19] S. Kachru and J. McGreevy, Supersymmetric three cycles and supersymmetry breaking, Phys. Rev. D 61 (2000) 026001 [hep-th/9908135] [INSPIRE].

[20] D. Lüst, S. Reffert, E. Scheidegger and S. Stieberger, Resolved toroidal orbifolds and their orientifolds, Adv. Theor. Math. Phys. 12 (2008) 67 [hep-th/0609014] [INSPIRE].

[21] K. Becker, M. Becker and A. Strominger, Five-branes, membranes and nonperturbative string theory, Nucl. Phys. B 456 (1995) 130 [hep-th/9507158] [InSPIRE]. 
[22] A. Sen, Non-BPS states and branes in string theory, in Advanced School on Supersymmetry in the Theories of Fields, Strings and Branes, (1999), pg. 187 [hep-th/9904207] [INSPIRE].

[23] A. Sen and B. Zwiebach, Tachyon condensation in string field theory, JHEP 03 (2000) 002 [hep-th/9912249] [INSPIRE].

[24] B. Heidenreich, M. Reece and T. Rudelius, The weak gravity conjecture and emergence from an ultraviolet cutoff, Eur. Phys. J. C 78 (2018) 337 [arXiv: 1712.01868] [INSPIRE].

[25] B. Heidenreich, M. Reece and T. Rudelius, Repulsive forces and the weak gravity conjecture, JHEP 10 (2019) 055 [arXiv: 1906.02206] [INSPIRE].

[26] J. Polchinski, Monopoles, duality and string theory, Int. J. Mod. Phys. A 19S1 (2004) 145 [hep-th/0304042] [INSPIRE].

[27] B. Heidenreich, M. Reece and T. Rudelius, Sharpening the weak gravity conjecture with dimensional reduction, JHEP 02 (2016) 140 [arXiv: 1509.06374] [INSPIRE].

[28] B. Heidenreich, M. Reece and T. Rudelius, Evidence for a sublattice weak gravity conjecture, JHEP 08 (2017) 025 [arXiv: 1606.08437] [INSPIRE].

[29] S. Andriolo, D. Junghans, T. Noumi and G. Shiu, A tower weak gravity conjecture from infrared consistency, Fortsch. Phys. 66 (2018) 1800020 [arXiv: 1802. 04287] [INSPIRE].

[30] D. Gaiotto, A. Kapustin, N. Seiberg and B. Willett, Generalized global symmetries, JHEP 02 (2015) 172 [arXiv: 1412.5148] [INSPIRE].

[31] R. Blumenhagen, D. Kläwer, L. Schlechter and F. Wolf, The refined swampland distance conjecture in Calabi-Yau moduli spaces, JHEP 06 (2018) 052 [arXiv: 1803.04989] [INSPIRE].

[32] G. Dvali, Black holes and large $N$ species solution to the hierarchy problem, Fortsch. Phys. 58 (2010) 528 [arXiv:0706.2050] [INSPIRE].

[33] M. Haack and J.U. Kang, Towards the 1-loop effective action of type IIB orientifolds, PoS (CORFU2016) 099 (2017) [INSPIRE].

[34] D. Robbins and T. Wrase, D-terms from generalized NS-NS fluxes in type-II, JHEP 12 (2007) 058 [arXiv: 0709.2186] [INSPIRE].

[35] R. Blumenhagen, M. Brinkmann and A. Makridou, Quantum log-corrections to swampland conjectures, JHEP 20 (2020) 064 [arXiv:1910.10185] [INSPIRE].

[36] H. Ooguri, E. Palti, G. Shiu and C. Vafa, Distance and de Sitter conjectures on the swampland, Phys. Lett. B 788 (2019) 180 [arXiv:1810.05506] [INSPIRE].

[37] T.W. Grimm and J. Louis, The effective action of type IIA Calabi-Yau orientifolds, Nucl. Phys. B 718 (2005) 153 [hep-th/0412277] [InSPIRE].

[38] P. Corvilain, T.W. Grimm and I. Valenzuela, The swampland distance conjecture for Kähler moduli, JHEP 08 (2019) 075 [arXiv: 1812.07548] [INSPIRE].

[39] C. Vittmann, The axion-instanton weak gravity conjecture and scalar fields, M.Sc. thesis, University of Heidelberg, Heidelberg, Germany (2018).

[40] T.W. Grimm, C. Li and E. Palti, Infinite distance networks in field space and charge orbits, JHEP 03 (2019) 016 [arXiv: 1811.02571] [INSPIRE].

[41] D.P. Lichtig, Testing swampland conjectures and the emergence proposal in a two-parameter Calabi-Yau model, M.Sc. thesis, Max-Planck-Institute for Physics, Munich, Germany (2019).

[42] G. Dall'Agata and M. Morittu, Covariant formulation of BPS black holes and the scalar weak gravity conjecture, JHEP 03 (2020) 192 [arXiv: 2001.10542] [INSPIRE]. 